$\begin{array}{cc}\text { Programa de Pós-Graduação em Engenharia de Produção - PPGEP } & \\ \text { Laboratório de Qualidade de Vida - LaQVida } & \text { REVISTA BRASILEIRA DE } \\ \text { Universidade Tecnológica Federal do Paraná - UTFPR } & \text { Ponta Grossa - PR - Brasil } \\ \text { v. 06, n. 03, jul./set. 2014, p. 199-205 } & \text { QUALIDADE DE VIDA }\end{array}$

DOI: $10.3895 /$ /S2175-08582014000300006

\title{
Qualidade de vida em voz de crianças eutróficas, com sobrepeso e obesas
}

\author{
Quality of life in voice eutrophic children, overweight and obese
}

\author{
Fernanda Pascotini \\ Universidade Federal de Santa Maria - UFSM - Santa Maria - Rio Grande do Sul - Brasil \\ fepascotini@hotmail.com \\ Leris Salete Bonfanti Haeffner \\ Universidade Federal de Santa Maria - UFSM - Santa Maria - Rio Grande do Sul - Brasil \\ leris.haeffner@gmail.com \\ Vanessa Veis Ribeiro \\ Universidade Federal de Santa Maria - UFSM - Santa Maria - Rio Grande do Sul - Brasil \\ Universidade Estadual do Centro-Oeste - UNICENTRO - Irati - Paraná - Brasil \\ vanessaribeirooo@hotmail.com \\ Carla Aparecida Cielo \\ Universidade Federal de Santa Maria - UFSM - Santa Maria - Rio Grande do Sul - Brasil \\ cieloca@yahoo.com.br
}

\section{RESUMO}

OBJETIVO: Verificar a percepção dos pais acerca da qualidade de vida em voz de crianças eutróficas, com sobrepeso e obesas.

MÉTODOS: Estudo transversal, observacional, analítico, de campo e quantitativo, do qual participaram 83 crianças, com idades entre 8 e 12 anos (média de 9,77 anos), sendo 37 (44,58\%) meninas e $46(55,42 \%)$ meninos, que passaram por uma avaliação antropométrica e cujos responsáveis responderam a um protocolo de triagem e ao protocolo Qualidade de Vida em Voz Pediátrico (QVV-P). Realizou-se análise estatística por meio do teste paramétrico ANOVA e adotou-se o nível de significância de 5\%.

RESULTADOS: As médias dos escores de qualidade de vida relacionada à voz nos domínios físico, socioemocional e total $(93,77 ; 97,21 ; 95,15$, respectivamente) estiveram dentro do esperado para crianças com vozes saudáveis. Observou-se que não houve diferença entre as médias de escores dos domínios físico, socioemocional e total, em função do estado nutricional das crianças, não sendo encontrada correlação entre o índice de massa corporal e os escores dos domínios de qualidade de vida relacionados à voz das crianças.

CONCLUSÕES: Conclui-se que no grupo de crianças estudadas o estado nutricional não interferiu na qualidade de vida relacionada à voz, encontrando-se dentro do esperado para crianças com vozes saudáveis.

PALAVRAS-CHAVE: Avaliação em saúde. Criança. Estado nutricional. Qualidade de vida. Voz. 


\section{ABSTRACT}

OBJECTIVE: Verify the parents' perception about the quality of life in voice obese children, overweight and obese.

METHODS: Cross-sectional study of analytical and quantitative field, attended by 83 children, aged between eight and 12 years (mean 9,77 years), and 37 (44,57\%) and 46 girls $(55,42 \%)$ boys who underwent an anthropometric assessment and whose parents answered a screening protocol and the protocol Pediatric Voice-Related Quality-of-Life (PVRQOL). We carried out statistical analysis using ANOVA parametric test, we adopted a significance level of 5\%.

RESULTS: The mean scores of quality of life related to voice in the physical, social-emotional domains, and total $(93,77 ; 97,21 ; 95,15$, respectively) were within the expected range for healthy children with voices. It was observed that there was no difference between the mean scores on the physical, social-emotional, and total fields, depending on the nutritional status of children, no correlation between body mass index and domain scores of quality of life related to voice being found children.

CONCLUSIONS: It follows that the group of children studied the nutritional status had no effect on voice-related quality of life, lying within the expected range for healthy children with voices.

KEYWORDS: Health eEvaluation. Child. Nutritional status. Quality of life. Voice.

\section{Introdução}

O conceito de qualidade de vida vem se modificando nos últimos anos, deixando de ser estruturado a partir do controle de sintomas, diminuição da mortalidade ou aumento da expectativa de vida dos indivíduos; e, passando a ser visto como um estado subjetivo de completo bem-estar (BEHLAU et al., 2009; RIBEIRO et al., 2013). O estudo da qualidade de vida tem razoável tradição na América Latina devido às condições socioeducativas da população e considera-se que ela exerça um papel muito importante nas condições de vida e saúde (BUSS, 2001; CARMO; CAMARGO; NEMR, 2006).

Observa-se que, nos últimos anos, houve um aumento das pesquisas que investigam a qualidade de vida de crianças e adultos em relação aos aspectos de saúde (POETA; DUARTE; GIULIANO, 2010; BOSELEY et al., 2006; RIBEIRO et al., 2013; RIBEIRO, PAULA, BEHLAU, 2014). A literatura aponta que o estado nutricional influencia diretamente a qualidade de vida em relação à saúde geral da criança (POETA; DUARTE; GIULIANO, 2010), porém, não foram encontrados estudos nacionais que tenham relacionado o estado nutricional à qualidade de vida em voz dessa população.

A voz faz parte dos componentes da linguagem oral e das relações interpessoais, portanto, produz impacto na qualidade de vida dos indivíduos (PENTEADO; BICUDO-PEREIRA, 2003; GRILLO; PENTEADO, 2005). Devido à possibilidade de deposição anormal de tecido adiposo nos órgãos fonoarticulatórios como faringe, laringe, língua, úvula e palato mole, e da interferência na função respiratória, acredita-se que a obesidade possa influenciar nos três níveis da produção vocal, respiratório, fonatório e ressonantal ou articulatório (CUNHA et al., 2009; TEIXEIRA et al., 2009). Além disso, o acúmulo de tecido adiposo na parede abdominal e torácica pode produzir um peso excessivo no diafragma, causando alteração em sua movimentação. Sendo assim, indivíduos obesos podem apresentar alteração do padrão respiratório, podendo comprometer a qualidade vocal (BORTOLOTTI; SILVA, 2005).

Compreender a percepção dos pais acerca da qualidade de vida em voz de seus filhos e relacioná-la ao índice de massa corporal pode fornecer dados importantes no que se refere à influência do estado nutricional sobre a utilização vocal diária, favorecendo o delineamento de ações multidisciplinares que abordem tais aspectos em crianças com distúrbios vocais. 
Diante do exposto, o presente estudo teve como objetivo verificar a percepção dos pais acerca da qualidade de vida em voz de crianças eutróficas, com sobrepeso e obesas.

\section{Métodos}

Trata-se de um estudo transversal, observacional, analítico, de campo e quantitativo. Inicialmente foram dados os esclarecimentos necessários ao responsável pela Secretaria Municipal de Educação, que assinou o Termo de Autorização Institucional (TAI). Os pais ou responsáveis pelos participantes receberam os esclarecimentos necessários e assinaram o Termo de Consentimento Livre e Esclarecido (TCLE).

Para selecionar a escola, foi realizado sorteio dentre as escolas municipais autorizadas pela Secretaria Municipal de Educação do município de Santa Maria/RS. Após a seleção da escola, todas as crianças foram convidadas a participar, sendo aplicados os critérios de seleção da amostra. Para selecionar a amostra, os pais responderam a um protocolo de triagem e as crianças passaram pela avaliação antropométrica. Foram incluídas crianças entre 8 e 12 anos, de ambos os sexos, com estado nutricional eutrófico, sobrepeso ou obesidade e que os responsáveis aceitaram participar da pesquisa e assinaram o TCLE. Os critérios de exclusão foram crianças que: apresentassem relato por parte dos responsáveis de afecções laríngeas, doenças neurológicas, gástricas ou respiratórias, estágio três ou superior de desenvolvimento puberal, respiração oral diagnosticada, relato de realização de terapia fonoaudiológica para voz ou aulas de canto prévias e índice de massa corporal (IMC) abaixo do peso ou desnutridas.

A mensuração do peso e da estatura foi realizada em tomada única. A pesagem foi realizada com as crianças descalças, vestindo roupas leves, em uma balança digital Magna $150 \mathrm{Kg}$ - G-Life. Para definição da estatura, as crianças foram colocadas descalças, em posição ereta, encostadas em uma superfície plana vertical, braços pendentes com as mãos espalmadas sobre as coxas, os calcanhares unidos e as pontas dos pés afastadas, formando ângulo de $60^{\circ}$, joelhos em contato, cabeça ajustada ao plano de Frankfurt e em inspiração profunda. Utilizou-se um estadiômetro portátil da marca Sanny, cujo cursor foi rebaixado lentamente até tocar o topo da cabeça em sua parte média, fixando-o, sem empurrá-la para baixo, e a leitura da régua foi realizada até o milímetro mais próximo, registrando-se o escore. A partir do peso e da altura, calculou-se o IMC, dividindo-se o peso em quilogramas pela altura em metros ao quadrado $\left(\mathrm{Kg} / \mathrm{m}^{2}\right)$. O diagnóstico do estado nutricional foi estabelecido individualmente, por meio do valor do IMC levado à distribuição nas curvas de Escore $\mathrm{Z}$. Considerou-se para crianças de 5 a 19 anos o escore $-2<\mathrm{Z} \leq+1$ eutrófica, escore $+1<\mathrm{Z} \leq+2$ sobrepeso e $\mathrm{Z}\rangle+2$ obeso (WHO, 2014).

Após a aplicação dos critérios de inclusão e exclusão, a amostra constituiu-se de 83 crianças, com idades entre 8 e 12 anos (média de 9,77 anos), sendo 37 (44,58\%) meninas e 46 (55,42\%) meninos, cujos pais responderam ao protocolo Qualidade de Vida em Voz Pediátrico (QVV-P) (BOSELEY et al., 2006; RIBEIRO, PAULA, BEHLAU, 2014). A média do IMC para os estados nutricionais foi: eutróficas 16,57, sobrepeso 20,46 e obesas 25,54.

O protocolo QVV-P é composto por 10 questões divididas nos domínios físico, socioemocional e total, que avaliam a percepção dos pais acerca do impacto de um possível problema de voz na qualidade de vida dos filhos (BOSELEY et al., 2006; RIBEIRO et al., 2013; RIBEIRO, PAULA, BEHLAU, 2014). Os pais foram orientados a marcar a alternativa correspondente à importância do problema de acordo com a escala: (1) não é um problema; (2) é um problema pequeno; (3) é um problema médio; (4) é um problema grande e (5) é um problema muito grande. Foi realizado cálculo específico proposto pelo instrumento para estabelecimento dos escores.

Os dados foram tabulados e as variáveis foram analisadas descritiva e estatisticamente por meio do teste paramétrico Anova. Adotou-se o nível de significância de 5\%. A presente pesquisa foi aprovada pelo Comitê de Ética em Pesquisa da Universidade Federal de Santa Maria (UFSM) sob o parecer $\mathrm{n}^{\mathrm{o}} 245.208$. 


\section{Resultados}

O grupo de crianças analisado foi composto por 83 crianças com idades entre 8 e 12 anos (média de 9,77 anos), sendo 37 (44,58\%) meninas e 46 (55,42\%) meninos. Na Tabela 1 é possível observar as médias dos escores de qualidade de vida em voz nos domínios físico, socioemocional e total.

\begin{tabular}{|c|c|c|c|}
\hline & Físico & Socioemocional & Total \\
\hline Média & 93,77 & 97,21 & 95,15 \\
\hline Mediana & 100,00 & 100,00 & 100,00 \\
\hline Desvio Padrão & 13,73 & 12,35 & 12,20 \\
\hline
\end{tabular}

Fonte: Autoria própria (2014).

A Tabela 2 mostra que não houve diferença entre as médias de escores dos domínios físico, socioemocional e total, em função do estado nutricional das crianças.

Tabela 2 - Análise das médias dos domínios de qualidade de vida em voz em função do estado nutricional de crianças

\begin{tabular}{|c|c|c|c|c|}
\hline Domínios QVV-P & Estadonutricional & Média & Desvio Padrão & $\mathrm{p}$-valor \\
\hline \multirow{3}{*}{ Físico } & EUT & 92,99 & 2,02 & \multirow{3}{*}{0,752} \\
\hline & SBP & 93,62 & 3,35 & \\
\hline & OBE & 95,83 & 3,17 & \\
\hline \multirow{3}{*}{ Socioemocional } & EUT & 95,87 & 1,80 & \multirow{3}{*}{0,501} \\
\hline & SBP & 98,16 & 3,00 & \\
\hline & OBE & 99,67 & 2,84 & \\
\hline \multirow{3}{*}{ Total } & EUT & 94,14 & 1,79 & \multirow{3}{*}{0,626} \\
\hline & SBP & 95,44 & 2,97 & \\
\hline & OBE & 97,36 & 2,81 & \\
\hline
\end{tabular}

* Valores estatisticamente significantes $(\mathrm{p} \leq 0,05)$ - Teste Anova.

Legenda: QVV-P: Qualidade de vida em voz pediátrico; EUT: eutrófico; SBP: sobrepeso; OBE: obeso.

Fonte: Autoria própria (2014).

Observa-se na Tabela 3 que não houve correlação entre os domínios de qualidade de vida em voz pediátricos com o índice de massa coporal das crianças.

Tabela 3 - Resultados da correlação entre os domínios de qualidade de vida em voz e o índice de massa corporal das crianças

\begin{tabular}{ccc}
\hline \multirow{2}{*}{ Domínios QVV-P } & \multicolumn{2}{c}{ IMC } \\
\cline { 2 - 3 } & corr & p-valor \\
\hline Físico & 0,002 & 0,979 \\
Sícioemocional & $-0,007$ & 0,948 \\
Total & $-0,003$ & 0,977 \\
\hline
\end{tabular}

* Valores estatisticamente significantes $(\mathrm{p} \leq 0,05)$ - Teste de Correlação de Pearson.

Legenda: QVV-P: Qualidade de vida em voz pediátrico; IMC=índice de massa corporal; corr=coeficiente de correlação. Fonte: Autoria própria (2014).

\section{Discussão}

Por não haver ainda um parâmetro de normalidade na literatura nacional, optou-se por utilizar o americano, no qual foram encontrados índices abaixo de 91,80 para meninos disfônicos e abaixo de 89,55 para meninas disfônicas (HARTNICK; VOLK; CUNNINGHAM, 2003). Considerando esse parâmetro, os indivíduos da presente pesquisa apresentaram média qualidade de vida em voz nos domínios físico, socioemocional e total $(93,77 ; 97,21 ; 95,15$, respectivamente) dentro do esperado para indivíduos com vozes saudáveis. 
Estudo nacional que aplicou o protocolo QVV-P em crianças disfônicas pré (M1) e pós (M2) terapia fonoaudiológica, mostrou escores semelhantes ao da presente pesquisa em M2 para os domínios físico, socioemocional e total (90,3; 96,8; 92,9, respectivamente) (RIBEIRO et al., 2013). Outra semelhança com o trabalho foi que os escores no domínio físico apresentaram-se mais baixos, seguido pelo total e socioemocional. Acredita-se que isso se deva ao fato da avaliação ser realizada pelos pais, que culturalmente veem as alterações vocais como passageiras, e não acreditam que elas possam interferir, quando isoladas, fisicamente e nas relações sociais das crianças (PENTEADO et al., 2007; RIBEIRO et al., 2013).

$\mathrm{Na}$ Tabela 2 foi mostrado que não houve diferença entre as médias de escores dos domínios físico, socioemocional e total, em função do estado nutricional das crianças. Tais dados também vão ao encontro de pesquisa nacional que comparou as avaliações objetivas e a percepção dos pais a respeito da qualidade de vida em voz dos filhos pré e pós-terapia, mostrando que, mesmo com a melhora significativa nos parâmetros objetivos, tais dados não eram encontrados na avaliação da qualidade de vida em voz realizada pelos pais (RIBEIRO et al., 2013). Outras pesquisas reforçam tais achados, mostrando que a falta de uma percepção acurada dos pais, no que se refere à voz infantil, faz com que a procura por auxílio profissional acabe ocorrendo apenas quando outros profissionais interferem, ou quando há outras patologias associadas, como respiratórias ou de aprendizagem (PENTEADO et al., 2007; FERNANDES; OLIVEIRA; BEHLAU, 2010; RIBEIRO et al., 2013).

Por outro lado, em pesquisa que foi relacionado os dados das análises perceptivoauditiva, acústica e de autopercepção vocal de crianças com e sem disfonia, foi mostrado que a autopercepção vocal negativa foi maior entre as crianças com disfonia, mostrando coerência na avaliação (OLIVEIRA et al., 2011). Sendo assim, questiona-se a inexistência na literatura de um instrumento de autoavaliação vocal que possa ser respondido pela própria criança, visto que a maior dificuldade da criança encontra-se na interpretação de um instrumento complexo, e não na autopercepção vocal. Tais dados poderiam aumentar a fidedignidade das pesquisas de qualidade de vida com crianças.

Na presente pesquisa, também, não foi encontrada correlação entre o IMC e os escores dos domínios de qualidade de vida em voz. Na literatura não foi encontrado estudo que relacionasse a obesidade à qualidade de vida em voz de crianças, porém, estudo que verificou a qualidade de vida geral por meio do instrumento PedsQL mostrou que o grupo de crianças obesas apresentou qualidade de vida inferior nos domínios físico, emocional, psicossocial e qualidade de vida geral, mostrando influência da obesidade na vida diária das crianças (POETA; DUARTE; GIULIANO, 2010). Tais dados não se aplicaram à qualidade de vida relacionada à voz das crianças, diferindo dos resultados encontrados na presente investigação.

Considerando a possibilidade de deposição anormal de tecido adiposo nos órgãos fonoarticulatórios como faringe, laringe, língua, úvula e palato mole, e da interferência do acúmulo de tecido adiposo na parede abdominal e torácica que produz alteração na movimentação do diafragma, acredita-se que a obesidade possa influenciar nos três níveis da produção vocal, respiratório, fonatório e ressonantal ou articulatório (CUNHA et al., 2009; TEIXEIRA et al., 2009). Visto que crianças são consideradas obesas quando possuem um IMC acima de 25 e a amostra da presente pesquisa está no limite inferior do grau de obesidade, pode-se inferir que o estado nutricional não está influenciando nos parâmetros vocais (OMS, 2006).

\section{Considerações finais}

Conclui-se que no grupo de crianças estudadas o estado nutricional não interferiu na qualidade de vida relacionada à voz, a qual se encontra dentro do esperado para crianças com vozes saudáveis.

\section{Agradecimentos}

Agradecemos ao CNPq e à CAPES pela concessão da bolsa de pesquisa. 


\section{Referências}

BEHLAU, M.; OLIVEIRA, G.; SANTOS, L. de M. A. dos; RICARTE, A. Validação no Brasil de protocolos de auto-avaliação do impacto de uma disfonia. Pró-Fono Revista de Atualização Científica, v. 21, n. 4, p. 326-232, out./dez. 2009. crossef

BORTOlOTTI, P.; SILVA M. A. A. Caracterização da voz de um grupo de mulheres com obesidade mórbida acompanhadas no Setor de Cirurgia Bariátrica da Irmandade Santa Casa de Misericórdia de São Paulo. Distúrbios da Comunicação, v. 17, n. 2, p. 149-160, ago. 2005.

BOSELEY, M. E.; CUNNINGHAM, M. J.; VOLK, M. S.; HARTNICK, C. J. Validation of the Pediatric Voice-Related Quality of Life Survey. Archives of Otolaryngology Head \& Neck Surgery, v. 132, n. 7, p. 717-720, jul. 2006. crossef

BUSS, P. N. Promoção de saúde e qualidade de vida. Ciência \& Saúde Coletiva, v. 5, n. 1, p. 134$142,2001$.

CARMO, R. D.; CAMARGO, Z.; NEMR, K. Relação entre qualidade de vida e autopercepção da qualidade vocal de pacientes laringectomizados totais: estudo piloto. Revista CEFAC, v. 8, n. 4, p. 518-528, out./dez. 2006. rossef

CUNHA, M. G. B.; PASSEROTTI, G. H.; WEBER, R.; ZILBERSTEIN, B. Caracterização da voz do indivíduo portador de obesidade mórbida. ABCD - Arquivos Brasileiros de Cirurgia Digestiva, v. 22, n. 2, p. 76-81, abr./jun. 2009.

FERNANDES, A. C. N.; OLIVEIRA, G.; BEHLAU, M. Desvantagem vocal na percepção do paciente e do informante substituto. In: CONGRESSO BRASILEIRO DE FONOAUDIOLOGIA, 18., 2010, Curitiba. Anais... Curitiba: Sociedade Brasileira de Fonoaudiologia, 2010.

GRILlO, M. H. M. M.; PENTEADO, R. Z. P. Impacto da voz na qualidade de vida de professore(a)s do ensino fundamental. Pró-Fono Revista de Atualização Científica, v. 17, n. 3, p. 321-330, set./dez. 2005. Crossef

HARTNICK, C. J.; VOLK, M.; CUNNINGHAM, M. Establishing normative voice-related quality of life scores within the pediatric otolaryngology population. Archives of Otolaryngology Head \& Neck Surgery, v. 129, n. 10, p. 1090-1093, out. 2003. Crossef

OLIVEIRA, R. C.; TEIXEIRA, L. C.; GAMA, A. C. C.; MEDEIROS, A. M. Análise perceptivoauditiva, acústica e autopercepção vocal em crianças. Jornal da Sociedade Brasileira de Fonoaudiologia, v. 23, n. 2, p. 158-163, 2011.

OMS. Organização Mundial da Saúde (OMS). Programas e projetos: obesidade e sobrepeso. 2006. Disponível em: <http://www.who.int/mediacentre/factsheets/fs311/en/índex.html>. Acesso em: 18 de fevereiro de 2013.

PENTEADO, R. Z.; BICUDO-PEREIRA, I. M. T. Avaliação do impacto da voz na qualidade de vida de professores. Revista da Sociedade Brasileira de Fonoaudiologia, São Paulo, v. 8, n. 2, p. 19-28, set./dez. 2003. 
PENTEADO, R. Z.; CAMARGO, A. M. D.; RODRIGUES, C. F.; SILVA, C. R.; ROSSI, D.; SILVA, J. T. C.; GONZALES, P.; SILVA, S. L. S. G. Vivência de voz com crianças: análise do processo educativo em saúde vocal. Distúrbios da Comunicação, v. 19, n. 2, p. 237-246, ago. 2007.

POETA, L. S.; DUARTE, M. F. S.; GIULIANO, I. S. B. Qualidade de vida relacionada à saúde de crianças obesas. Revista da Associação Médica Brasileira, v. 56, n. 2, p. 168-172, 2010. rossef

RIBEIRO, L. L.; PAULA, K. M. P.; BEHLAU, M. Qualidade de vida em voz na população pediátrica. CoDAS, v. 26, n. 1, p. 87-95, jan./fev 2014.

RIBEIRO, V. V.; LEITE, A. P. D.; FILHO, L. L.; CIELO, C. A.; BAGAROLLO, M. F. Percepção dos pais sobre a qualidade de vida em voz e evolução clínica de crianças disfônicos pré e pósterapia fonoaudiológica em grupo. Distúrbios da Comunicação, v. 25, n. 1, p. 81-90, abr. 2013.

TEIXEIRA, V. S. S.; FONSECA, B. C. A.; PEREIRA, D. M.; SILVA, B. A. K.; REIS, F. A. Avaliação do efeito da obesidade infantil e a do adolescente sobre as propriedades ventilométricas e força muscular do sistema respiratório. ConScientia e Saúde, v. 8, n. 1, p. 35-40, 2009.

WHO - WORLD HEALTH ORGANIZATION. Obesity and overweight. 2014. Disponível em: <http://www.who.int/mediacentre/factsheets/fs311/en>. Acesso em: 19 June 2014. 